\title{
Knorpelveränderungen in den Fußwurzelgelenken während eines Ultramarathonlaufs
}

Schütz UH et al. MRI Cartilage Assessment of the Subtalar and Midtarsal Joints During a Transcontinental Ultramarathon - New Insights into Human Locomotion. Int J Sports Med 2018; 39: 37-49

Marathonläufe und sogar Ultramarathonläufe - über eine Distanz $>42 \mathrm{~km}$ - werden immer beliebter. Verschiedene Studien haben gesundheitliche Auswirkungen solcher Extrembelastungen untersucht, sich dabei aber meistens auf Herz-Kreislauf-System, Atmung und Skelettmuskulatur konzentriert. Wie solche Laufstrecken die Gelenke, insbesondere den Gelenkknorpel, beeinflussen, haben Mediziner aus UIm und Minneapolis überprüft.

Uwe Schütz und seine Kollegen haben dazu 22 Teilnehmer (20 Männer) des 2. Transeuropalaufs von April bis Juni 2009 in eine prospektive Laborstudie aufgenommen; 13 Teilnehmer (12 Männer) nahmen bis zum Ende teil. Bei dem Lauf handelte es sich um einen Etappen-Ultramarathon, der in 64 Tagesetappen ohne dazwischengeschaltete Ruhetage über 4486 km von Süditalien bis zum Nordkap führte.

Bei den Sportlern untersuchten die Wissenschaftler mithilfe eines mobilen, über die gesamte Strecke mitgeführten MRTGeräts die Veränderungen des Knorpels in den Fußwurzelgelenken im Verlauf des Rennens und verglichen die Daten mit den Ausgangswerten vor Rennbeginn.

Die MRT-Untersuchung beider Füße erfolgte

- zum Zeitpunkt t0, innerhalb von 4 Tagen vor Rennbeginn,

- zum Zeitpunkt t1, nach einer Strecke von ca. $1200 \mathrm{~km}$ und

- zu den Zeitpunkten t2-t4, nach jeweils ca. 900 km Laufstrecke.

Dabei bestimmten Schütz et al. die T2* Relaxationszeiten im Knorpel des subtalaren Gelenks (Articulatio talocalcanea) und der mediotarsalen Gelenke (Articulatio 


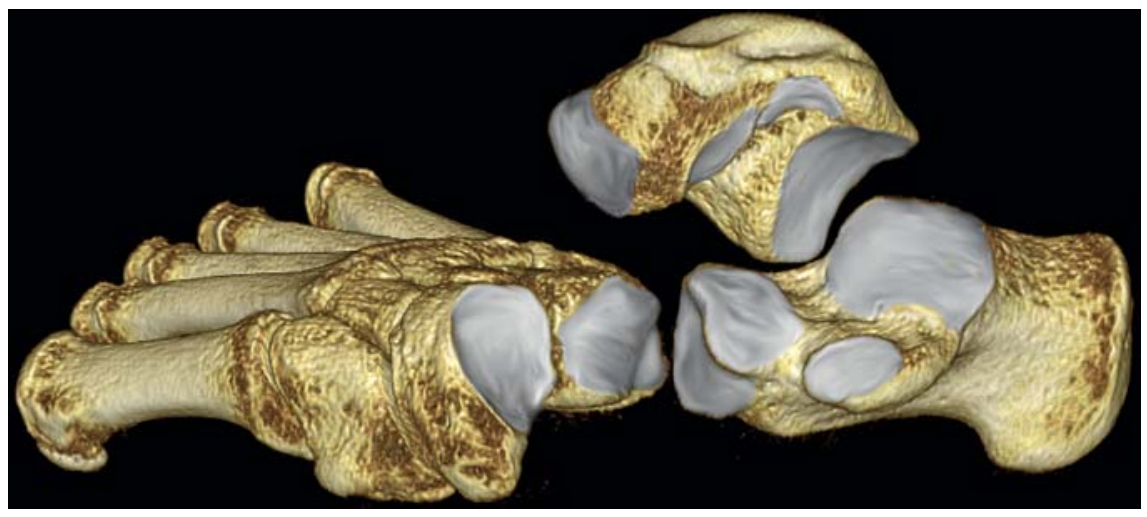

3-dimensionale Darstellung der regions of interest. Quelle: Schütz UHW et al. MRI Cartilage Assessment of the Subtalar and Midtarsal Joints During a Transcontinental Ultramarathon New Insights into Human Locomotion. Int J Sports Med 2018; 39: 37-49.

talonaviculare, Articulatio calcaneocuboidea). Ein Anstieg der T2*-Relaxationszeiten steht für eine zunehmende strukturelle Anisotropie der Kollagenmatrix des Gelenks und eine Zunahme des freien Wassers, das aus seiner chemischen Bindung an die Proteoglykankomponente abdissoziiert.

Die Auswertung ergab vor Rennbeginn eine durchschnittliche Knorpeldicke im subtalaren Gelenk von 2,6-3,1 mm und in den mediotarsalen Gelenken von 1,92,7 mm. Diese Werte veränderten sich im Verlauf des Rennens nicht wesentlich.

Die T2*-Relaxationszeiten nahmen über die Zeit in nahezu allen beurteilten Regionen des Gelenkknorpels im subtalaren Gelenk zu; auf der Strecke von 1200 2400 km waren es im Mittel 20,9\% im Vergleich zum Wert vor dem Rennen. Während der 2. Hälfte des Rennens folgte dann eine erneute Abnahme der Zeiten um im Mittel 6\%; das entsprach 28,5\% der anfänglichen Zunahme. In den mediotarsalen Gelenken fanden sich ähnliche Verhältnisse: Einer Zunahme der T2* -Zeiten um 26,3\% während des ersten Rennabschnitts folgte eine Verminderung um $4,2 \%$, entsprechend $16,0 \%$ der anfänglichen Zunahme.

Dabei fand sich interessanterweise eine asymmetrische Verteilung dieser Parameter: Sie war im Mittel im rechten Fuß signifikant stärker ausgeprägt als im linken.
Die T2*-Relaxationszeit korrelierte mit der zurückgelegten Gesamtlaufstrecke und der gelaufenen Zeit. Körpergewicht und Body-Mass-Index der Teilnehmer zeigten dagegen keine entsprechenden Assoziationen.

Es fand sich lediglich 1 manifeste osteochondrale Läsion mit begleitendem Knochenmarködem am Os naviculare (Grad 1 nach Outerbridge). Die Läsion blieb dabei stabil, aber die T2*-Zeit nahm im Verlauf zu.

\section{FAZIT}

Eine extreme körperliche Belastung ist also mit signifikanten Veränderungen des Aufbaus des belasteten Knorpels verbunden, fassen die Autoren zusammen. Diese Veränderungen sind zeitnah, quasi „in vivo“, in der mobilen MRT nachweisbar. Weiterhin kommt es nach diesen Daten anscheinend auch unter andauernder Belastung zu einer zumindest partiellen Adaptation der Chondrozyten, indem sie die Proteoglykan- und Kollagensynthese in Anpassung an die erhöhte Anforderung steigern.

Dr. Elke Ruchalla, Bad Dürrheim 